\title{
Generation of induced pluripotent stem cells by a polycistronic lentiviral vector in feeder- and serum- free defined culture
}

\begin{abstract}
Induced pluripotent stem cells (iPSCs) have great potentials for regenerative medicine. However, serious concerns such as the use of the viral-mediated reprogramming strategies and exposure of iPSCs to animal products from feeder cells and serumcontaining medium have restricted the application of iPSCs in the clinics. Therefore, the generation of iPSCs with minimal viral integrations and in non-animal sourced and serum-free medium is necessary. In this report, a polycistronic lentiviral vector carrying Yamanaka's factors was used to reprogram mouse fibroblasts into iPSCs in feeder- and xeno-free culture environment. The generated iPSCs exhibited morphology and selfrenewal properties of embryonic stem cells (ESCs), expression of specific pluripotent markers, and potentials to differentiate into the three-major distinct specialized germ layers in vitro. The iPSCs were also shown to have the potential to differentiate into neural precursor and neurons in culture, with greater than $95 \%$ expression of nestin, Pax6 and $\beta$ III-tubulin. This body of work describes an alternative method of generating iPSCs by using polycistronic lentiviral vector that may minimize the risks associated with viral vector-mediated reprogramming and animal derived products in the culture media.
\end{abstract}

Keyword: Feeder-and serum-free; Neural precursor and neuron cells; Polycistronic lentiviral vector; iPSCs 
